\title{
Effect of iterative reconstructions in low dose computed tomography
}

\author{
TBS Buxi, Anurag Yadav, Kishan Singh Rawat, Samarjit Singh Ghuman \\ Department of CT \& MRI, Sir Gangaram Hospital, New Delhi, India \\ Correspondence: Anurag Yadav. Address: Consultant Radiologist, Department of CT \& MRI, Sir Gangaram Hospital, New \\ Delhi-110060, India. E-mail: anu_nalin@hotmail.com
}

Received: February 23, 2014

Accepted: April 24, 2014

Online Published: May 21, 2014

DOI : $10.5430 /$ jbgc.v4n3p1

URL: http://dx.doi.org/10.5430/jbgc.v4n3p1

\section{Abstract}

Objectives: To assess the patient radiation dose profiles in routine body image acquisitions when using iterative reconstruction (IR) methods and compare them with filtered back projection technique.

Material and methods: Iterative dose profiles of 986 patients - Thorax (317), Abdomen/Pelvis (540), Thorax/ Abdomen/Pelvis (129) in routine clinical practice have been evaluated and effective patient dose estimated from dose length product (DLP) Values. Within the evaluated samples, distribution of CT Dose Index (CTDI) and DLP values were calculated.

Results: In Thorax, average DLP delivered was $286.30 \mathrm{mGy.cm}$ which corresponded to an effective patient dose of 4.008 $\mathrm{mSv}$. In Abdomen/Pelvis group, average value of DLP was $495.5 \mathrm{mGy}$, estimated dose was $7.43 \mathrm{mSv}$. In trunk study group average DLP was $570.21 \mathrm{mGy}$ which corresponded to an effective dose of $8.55 \mathrm{mSv}$.

Conclusion: Substantial dose reduction is achieved with iterative techniques. In 986 patients scans evaluated, average dose reduction of 33.2\% in Thorax, 32.455\% in Abdomen/Pelvis and $49.706 \%$ in trunk examinations were estimated.

\section{Key words}

Iterative reconstruction, IDose4, Dose reduction, Low dose CT imaging

\section{I ntroduction}

CT technology and its applications have advanced by leaps and bounds over the years. Increased use of CT and its applications have caused deep concern regarding radiation. In fact, radiation dose has been the most debated topic with Multi row Detector CT (MDCT) imaging. A comprehensive approach to radiation dose management should be focused on delivering the right quantity and quality of radiation, as and when required. Filtered back projection (FBP) has been the standard CT reconstruction technique for several years, almost since its inception, primarily due to its ability to practically reconstruct high quality images with the available computing resources ${ }^{[1]}$. However, the limitations of FBP reconstruction have become more apparent as dose levels have been pushed to new lows in the context of use of ionizing radiation to as low as reasonably achievable (ALARA) ${ }^{[1]}$. If ultra-low dose is used in FBP, the images are excessively noisy, which may obscure clinically significant anatomy or pathology. The second limitation is image artifacts due to photon starvation. 
Iterative reconstruction (IR) technique recently introduced by several CT vendors is primarily aimed to overcome these inherent limitations of FBP imaging. IR is supposed to be designed not only to reduce radiation dose to substantially lower range but also improve image quality ${ }^{[1]}$. Currently, CT imaging technology is clearly in a transition period from FBP to IR techniques. Most of the CT vendors have already launched iterative based low dose imaging techniques which is available for patients imaging with their newest products or available as an upgrade to the existing CT scanner installations. The degree of dose reduction achieved with their IR technologies varies with CT vendors, with a maximum claim of $80 \%$ dose reduction claimed by Philips Healthcare ${ }^{[1]}$.

CT radiation dose characteristics with new generation iterative techniques and its impact on reducing radiation dose to patients in body imaging have been evaluated in this study.

\section{Objectives}

- To assess the patient radiation dose profiles in routine body image acquisitions when using IR methods.

- To evaluate the extent of dose savings achieved with IR methods in comparison with reported dose values with conventional FBP methods for various anatomical regions of the body.

- Evaluate the variation in CT Dose Index (CTDI) and dose length product (DLP) in single cumulative studies of the entire thorax, abdomen and pelvis (trunk) examination compared to individual split examinations of Thorax scanning and Abdomen/Pelvis examinations.

\section{Material and methods}

Dose summary tables from patient case studies performed for a period of approximately three months (from 1st July 2012 to 20th September 2012) in the Department of CT Imaging at Sir Ganga Ram Hospital, New Delhi. These were retrospectively recorded as dose profile input data. The case studies were acquired on Philips 128 Slice Ingenuity CT scanner with iterative reconstructions technique (iDose4) enabled data acquisitions. These were obtained for evaluation from the data base available within the Intelli space Portal workstations. Various body anatomy datasets were classified into the following categories: (1) individual Thorax studies; (2) combined Abdomen and Pelvis studies; and (3) combined cases studies of thorax, abdomen and pelvis (trunk). Entire patient case studies falling in these three categories during the above period were included in the database for evaluation, with the exclusion of infants and paediatric patients. CTDI and DLP values reported by the scanner for individual \& combined studies (trunk) have been compared with reported values of typical radiation dose values ${ }^{[2]}$. Effective radiation dose for the examinations were derived from DLPs values multiplying them with anatomy/organ specific conversion factors $(\mathrm{k})^{[2]}$. A total of 986 patient data had been included for analysis and evaluation. Readings of the highest value of CTDI and DLP within the acquisition series summary table were recorded.

\subsection{Derivation of effective radiation dose in millisieverts from CTDI / DLP values}

Most of the CT scanners display and programmed to automatically archive radiation dose parameters corresponding to the scan parameters set for every exposure protocol after each study. These measures are the CTDI vol and DLP. CTDI represents the average dose profile of the slice in a given image volume, measured and calculated as a mathematical integral, under the radiation dose profile of a single rotation scan that will produce one tomographic image at a fixed table position ${ }^{[2]}$. DLP is the product of the CTDI and the length of the scanned section which indicates the total dose delivered per acquisition ${ }^{[2]}$. The value of DLP is, by convention, stated in mGy.cm. CTDI and DLP values have been taken as the primary reporting factors in dose estimation in this study ${ }^{[2]}$. The effective radiation dose can be measured by a method proposed by the European Working Group for Guidelines on Quality Criteria in CT ${ }^{[3]}$ or according to the guidelines set by the AAPM Task Group 23 of the diagnostic Imaging Council CT Committee, January 2008 - "The measurement, 
Reporting and management of Radiation Dose in CT” (AAPM-96) ${ }^{[2]}$. The Radiation measurements followed in estimating the dose profile mentioned in this study are in accordance with the AAPM-96 guidelines ${ }^{[2]}$.

Effective radiation dose is calculated from the product of DLP and an organ weighting factor corresponding to the anatomical region examined ${ }^{[2]}$. Various anatomical regions have normalized weighting factors $(\mathrm{k})$ derived based on the radiation sensitivity profiles of the attenuated anatomical structures. The $\mathrm{k}$ value for thorax it is $0.14 \mathrm{mGy} . \mathrm{cm}$ and for abdomen and pelvis it is $0.15 \mathrm{mGy} . \mathrm{cm}^{[2]}$.

Table 1. Organ weighing factor k-values for effective dose estimation as reported in AAPM report 96

\begin{tabular}{llllll}
\hline Region of body & \multicolumn{5}{c}{$\mathbf{K}\left(\mathbf{~} \mathbf{~ S v ~} \mathbf{~ G y ~}^{\mathbf{- 1}} \mathbf{~ c m}^{\mathbf{- 1}} \mathbf{~}\right.$} \\
\hline & 0 year old & 1 year old & 5 year old & 10 year old & Adult \\
Head and neck & 0.013 & 0.0085 & 0.0057 & 0.0047 & 0.0031 \\
Neck & 0.017 & 0.0067 & 0.0040 & 0.0032 & 0.0021 \\
Chest & 0.039 & 0.026 & 0.018 & 0.0079 & 0.0059 \\
Abdomen\& pelvis & 0.049 & 0.030 & 0.020 & 0.015 & 0.015 \\
Trunk & 0.044 & 0.028 & 0.019 & 0.014 & 0.015 \\
\hline
\end{tabular}

Table 2. Typical effective dose values as reported in AAPM report 96

\begin{tabular}{llll}
\hline Non CT Typical Effective Dose values (mSv) & \multicolumn{3}{l}{ CT Typical Effective Dose values (mSv) } \\
\hline Head radiograph & $<0.1$ & Head CT & $1-2$ \\
Dental bitewing & $<0.1$ & Chest CT & $5-7$ \\
Chest radiograph & $0.1-0.2$ & Abdomen CT & $5-7$ \\
Mammogram & $0.3-0.6$ & Pelvis CT & $3-4$ \\
Lumbar spine radiograph & $0.5-1.5$ & Abdomen\&pelvis CT & $8-14$ \\
Barium enema exam & $3-6$ & Coronary artery calcium CT & $1-3$ \\
Coronary angiogram & $5-10$ & Coronary CT angiography & $1-3$ \\
Thallium myocardial perfusion & $35-40$ & Coronary CT angiography & $5-15$ \\
\hline
\end{tabular}

\subsection{Outcome measures}

As mentioned, CTDI, DLP values and effective dose estimated in $\mathrm{mSv}$ from DLP values were the major outcome measures. The effective dose was estimated by using conversion factors (k) mentioned in the AAPM report $96^{[2]}$.

Conversion factors $(\mathrm{k})$ used for analysis: Thorax $=0.014$; Abdomen $=0.015$; Abdomen and Pelvis $=0.015$; Thorax, Abdomen and Pelvis (trunk) $=0.015^{[2]}$

Effective dose $=\mathrm{DLP} \times \mathrm{k}^{[2]}$.

Table 3. Effective dose conversion factor $\mathrm{k}$ for various anatomical regions

\begin{tabular}{ll}
\hline Region of Body & $\mathbf{k}\left(\mathbf{m S v} \mathbf{~ m G y}-{ }^{1} \mathbf{c m}^{-\mathbf{1}}\right)$ \\
\hline Thorax & 0.014 \\
Abdomen \& Pelvis & 0.015 \\
Trunk (Thorax, abdomen and Pelvis) & 0.015 \\
\hline
\end{tabular}

\subsection{Clinical data acquisition for thorax}

As this is a retrospective analysis using the acquired clinical data samples, optimizations of the acquisition protocols to deliver the ideal parameters were beyond the scope of evaluation of the study. In order to enhance image quality and deliver the right exposure parameters, the scanner protocols incorporated the following dose modulation and dose optimization features along with the iDose4 IR technique. 
- ACS: Automatic current selection in which the scanner automatically suggests the tube current required for a patient scan basing the body attenuation profile inputs it received from the Surview (Scanogram) scan. With the use of ACS, the scanner either suggests a lower mAs or higher mAs depending on the attenuation profile of the patient surviewed.

- D-Dom: The dynamic/angular modulation is based on the attenuation profile of the anatomical region within the course of each helical rotation, the scanner automatically adjusts the tube current to best suit (tube current variation implied in Anterior-Posterior or Lateral position within the course of helical rotations).

Thorax scanning data of three hundred and seventeen patients were evaluated. These data sets were acquired with applications of moderate degree of IRs to compensate and remove noise and enhance image quality. Within the available iteration scale of 1-6 iterative compensations (1-mild compensation at high dose; 6-maximum compensation at lowest dose) the protocols were archived with iterative scaled factor 3.0 \& 4.0 levels.

The length of acquisitions (superior-inferior- $\mathrm{Z}$ axis coverage) were found to mildly varying within the samples analyzed. The variability was mostly observed in the inferior end point of the acquisitions. Mostly the thorax scan covered through lung apex to the diaphragm or up to the mid portion of liver. However, in some of the samples, the lower third portion of the liver and upper portion of kidneys also got covered. Sufficient margins in both caudal and cranial directions for coverage had been implemented, which resulted in maximizing DLPs estimated out of the sample analysis.

\subsection{Clinical data acquisition for abdomen and pelvis}

The scanner protocols for Abdomen and Pelvis imaging incorporated the following dose modulation and dose optimization features along with iDose4 IR technique.

- ACS

- Dose Right Z-Dom: Tube current modulation in the Z-direction. Using the anatomical attenuation profile within the course of helical tube rotations, the scanner automatically adjusts the tube currents with the attenuation profile readings it obtained from the immediate previous rotations in Z- direction.

Abdomen and Pelvis scanning data of five hundred and forty patients was evaluated. These data sets were acquired with applications of moderate degree of IRs (iDose4) and iterative scaled factor 4.0 levels.

The length of acquisitions (superior-inferior; Z-axis coverage) was nearly identical within the populations analyzed. No apparent coverage variability was observed between the male and female populations. The Abdomen and Pelvis scans were from above the diaphragm to three inch below the symphysis pubis, covering the entire pelvis. In some of the samples, the upper margin of the scan was just the level of diaphragm.

\subsection{Clinical data acquisition for thorax, abdomen and pelvis (trunk)}

The scanner protocols for combined Thorax, Abdomen and Pelvis imaging incorporated ACS and Dose right Z-Dom dose features for dose optimization along with iDose4 IR technique. Examination data of one hundred and twenty nine patients was evaluated. Moderate iterative compensation was applied; the scanning protocols were set with iterative scale factor 4.0 .

The length of acquisitions (superior-inferior; Dose right Z-axis coverage) was almost identical within the populations analyzed. The scans covered from apex of lungs to three inch below the symphysis pubis, covering the entire thorax, abdomen and pelvis areas. 


\section{Results}

\subsection{Thorax scans}

The lowest CTDI extracted from the thorax sample was $2.95 \mathrm{mGy}$, the highest value was $17.42 \mathrm{mGy}$ and the average value of CTDI derived was $7.42 \mathrm{mGy}$. The lowest DLP among the sample was $108.3 \mathrm{mGy} . \mathrm{cm}$, the highest value of DLP was $726.8 \mathrm{mGy} . \mathrm{cm}$ and the average DLP was $286.3 \mathrm{mGy} . \mathrm{cm}$. These values corresponded to an average effective patient dose of $4.008 \mathrm{mSv}$.

The distribution of CTDI and DLP among the population analyzed was as below (see Figures 1-3)

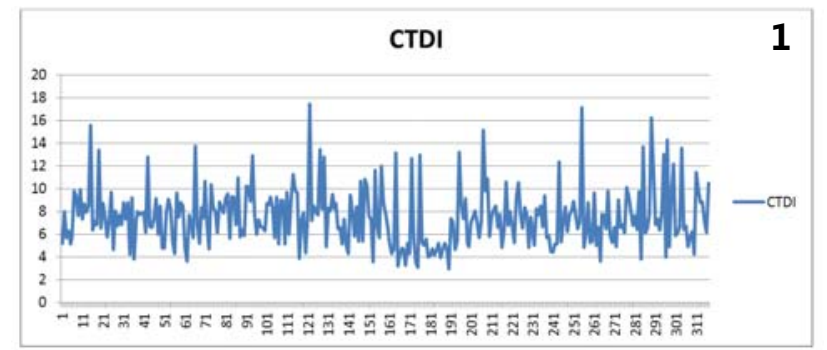

Figure 1. Graph representing the distribution of CTDI values (mGy) against samples of three hundred and eleven Thorax scans.

Figure 2. Graph representing the distribution of DLP (mGy.cm) values in Thorax scans.

Figure 3. Effective dose plotted $(\mathrm{mSv})$ against expected average dose in Thorax scans.

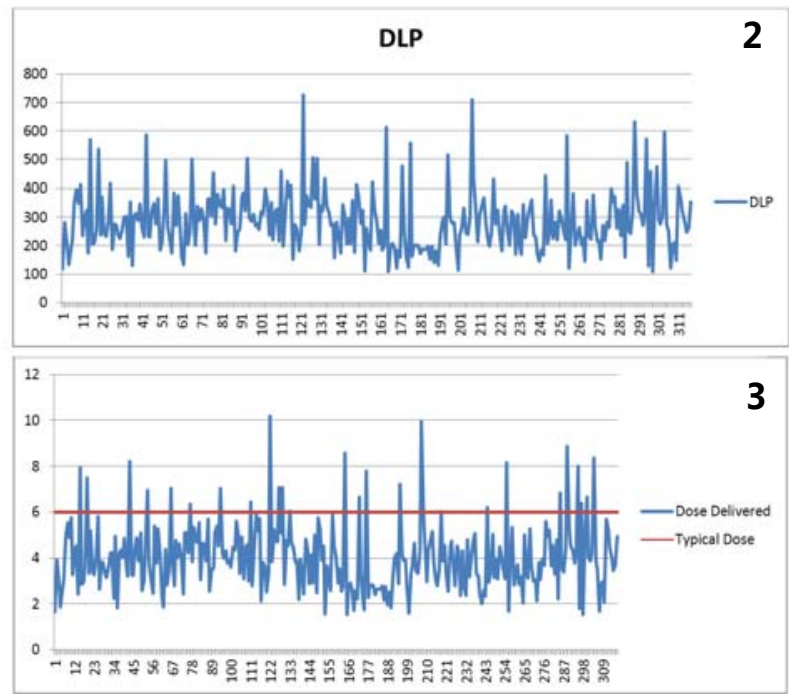

\subsection{Abdomen and Pelvis scans}

The lowest CTDI value extracted from the Abdomen and Pelvis sample was $2.9 \mathrm{mGy}$, the highest was $24.16 \mathrm{mGy}$ with an average CTDI value of $9.70 \mathrm{mGy}$. The lowest DLP among the examinations was noted as $127 \mathrm{mGy} . \mathrm{cm}$, the highest dose was $1230 \mathrm{mGy} . \mathrm{cm}$ and the its average was estimated as $495.5 \mathrm{mGy} . \mathrm{cm}$. Average effective dose delivered has been derived as $7.43 \mathrm{mSv}$ (see Figures 4-6).

Figure 4. Graph representing the distribution of CTDI values (mGy) against samples of five hundred and forty Abdomen and Pelvis scans.

Figure 5. Graph representing distribution of DLP values (mGy.cm) in Abdomen and Pelvis scans.

Figure 6. Effective dose (mSv) against expected average dose in Abdomen and Pelvis scans.
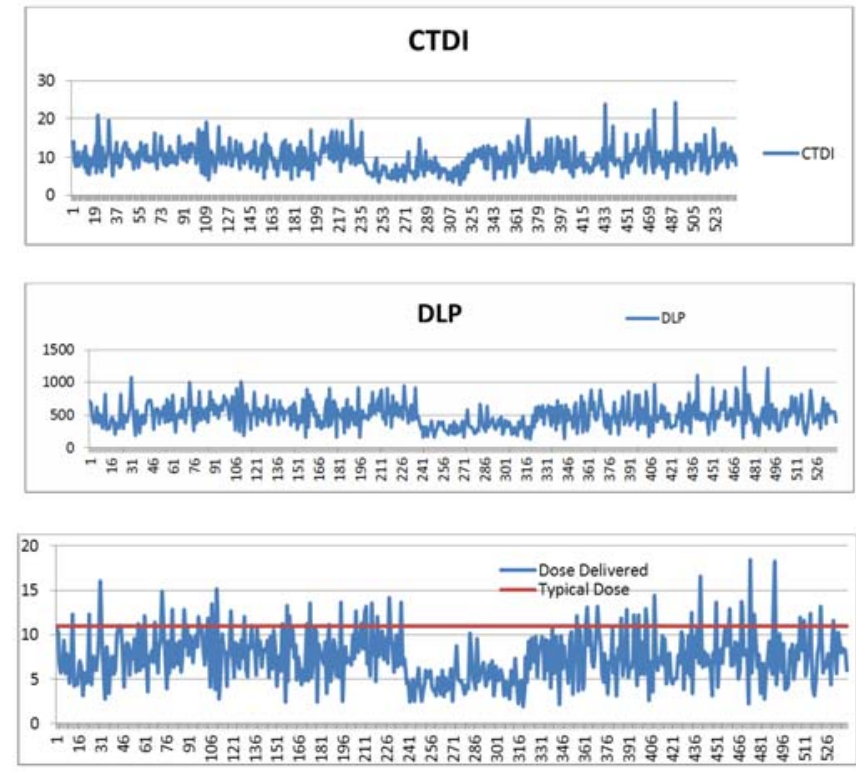


\subsection{Thorax, abdomen and pelvis scans (trunk)}

The lowest CTDI extracted from the thorax, abdomen and pelvis (trunk) population sample was 3.58 mGy, the highest was noted as $19.75 \mathrm{mGy}$ and the average reading was 8.43 mGy (see Figures 7-9).

The lowest DLP in the sample was noted as $172.4 \mathrm{mGy} . \mathrm{cm}$, the highest 1,414.5 mGy.cm and an average was estimated as $570.21 \mathrm{mGy} . \mathrm{cm}$. Average effective dose delivered was $8.55 \mathrm{mSv}$ (see Figures 10, 11).

Figure 7. Graph representing distribution of CTDI values (mGy) in combined studies of Thorax, Abdomen \& Pelvis (trunk) scans of 129 patients.

Figure 8. Graphs representing distribution of DLP (mGy.cm) values in combined studies of Thorax, Abdomen \& Pelvis (trunk) scans.

Figure 9. Graphs representing effective dose (mSv) against average effective dose in Thorax, Abdomen and Pelvis scans (trunk).

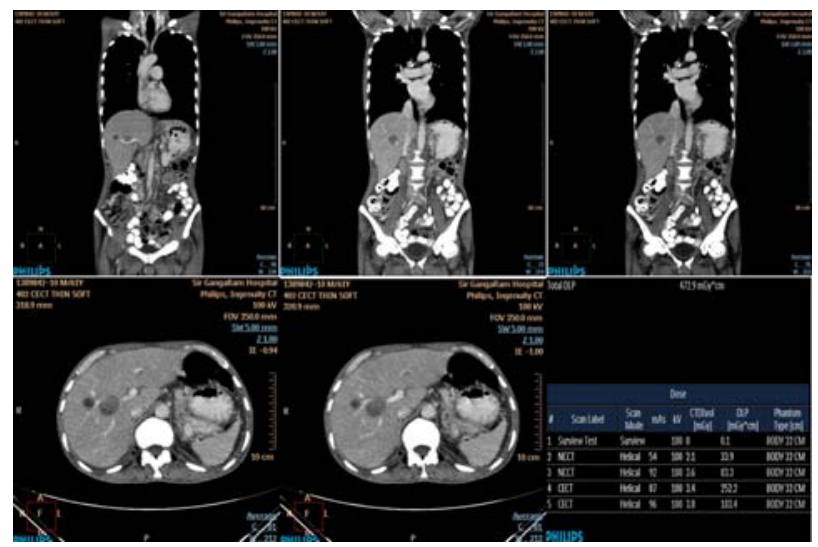

Figure 10. Thorax, abdomen and pelvis image reconstructed with IR technique, clearly demonstrating hepatic lesions. The CTDI delivered is $3.8 \mathrm{mGy}$ and total DLPS is 472.9 mGy.cm
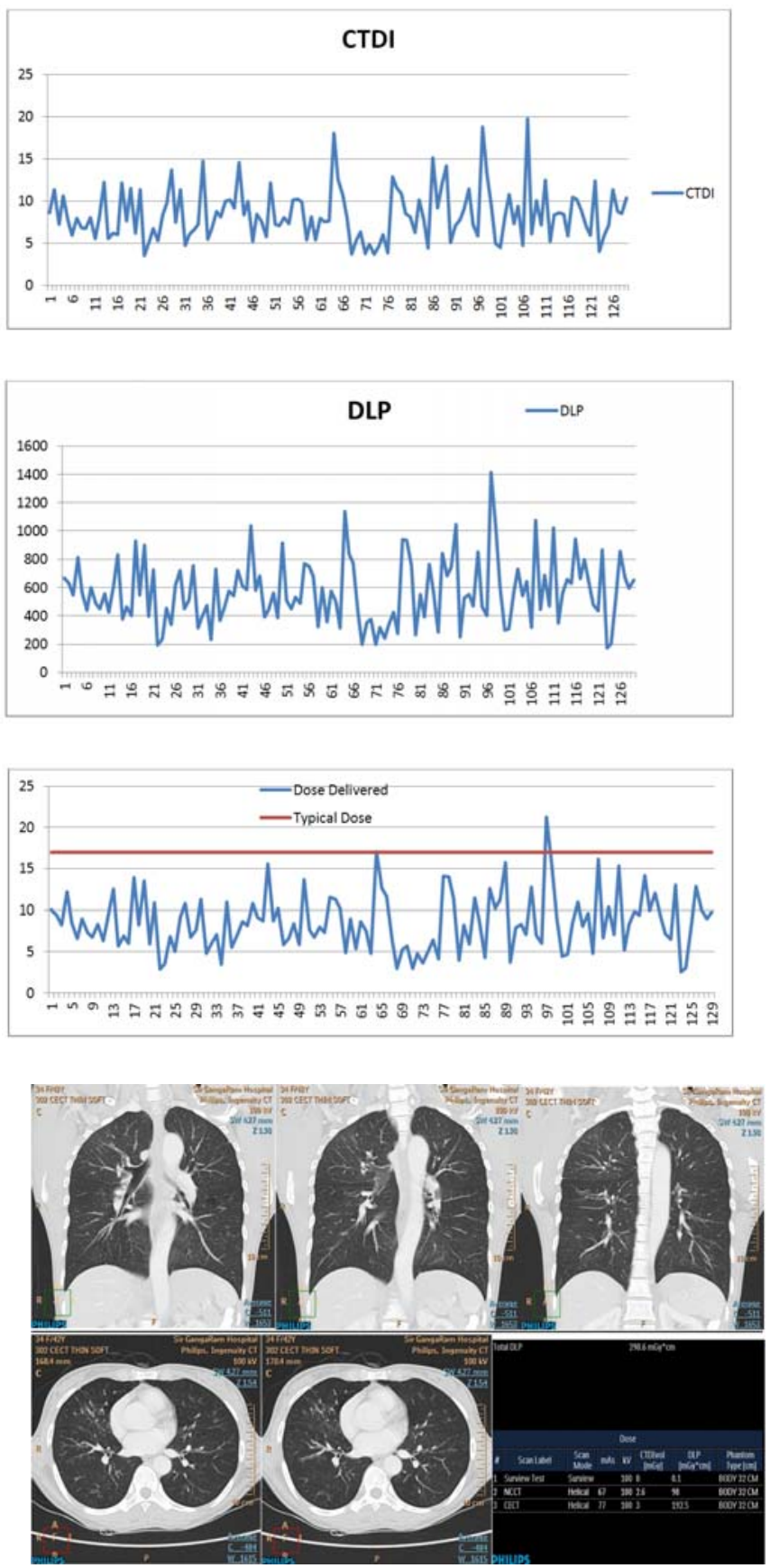

Figure 11. Thorax, abdomen and pelvis image reconstructed with IR technique, clearly demonstrating hepatic lesions. The CTDI delivered is $3 \mathrm{mGy}$ and total DLPS is 290.6 mGy.cm 


\section{Discussion}

With newer developments with IR technology, CT manufactures claim up to $80 \%$ dose reduction while maintaining diagnostic image quality ${ }^{[1]}$. IR is definitely considered a breakthrough in CT imaging, which is designed to provide equivalent diagnostic image quality with the similar appearance as full-dose images, at a fraction of the radiation dose. Similar to FBP techniques, these technologies also work in concert with other dose optimizing tools including filters, detectors and collimators ${ }^{[1]}$. Automatic current selection (ACS), which adapts tube current to patient size, dose modulation tools such as Z-axis Dose Modulation (Z-DOM) and Dynamic (Angular) Dose Modulation (D-DOM) to automatically adjust dose delivered to the patient, compensating for individual physiology and optimizing dose by anatomic region are also used in conjunction.

Rehani MM et al. ${ }^{[4]}$ in the editorial Radiation Doses in Computed Tomography suggested alternative examinations to control the increasing doses of radiation such as reconsidering the use of computed tomography in follow up of testicular malignancy, low dose computed tomography for chest and using ultrasound and magnetic resonance imaging for imaging the abdomen although it appears attractive in terms of speed and cost to perform the whole study involving chest and abdomen with computed tomography. For head and neck pathology magnetic resonance imaging has superseded computed tomography and often offers an alternative to examining the abdomen and pelvis. Computed tomography has limited utility and lack of cost-effectiveness for clinical staging in patients with suspected carcinoma of prostate. They also recommended shielding the superficial radiosensitive organs such as the breast, the lens of the eye and the thyroid, and the testes in computed tomography of the thorax, head, and pelvis respectively using thinly layered bismuth radio-protective latex or leaded garments, without affecting the display of other deeper structures, especially in children.

Hara A et al. ${ }^{[5]}$ evaluated image noise, low-contrast resolution, image quality and spatial resolution of adaptive statistical iterative reconstruction in low-dose body CT using adaptive statistical iterative reconstruction and showed that low-dose CT with adaptive statistical iterative reconstruction was associated with CT dose index reductions of 32\%-65\% compared with routine imaging and had the least noise both quantitatively and qualitatively $(p<.05)$ and that low-dose CT with adaptive statistical iterative reconstruction and routine-dose CT had identical results for low-contrast resolution and nearly identical results for overall image quality though routine-dose CT had better spatial resolution than low-dose CT $(p=$ $.004)$.

Lutterman AC et al. ${ }^{[6]}$ examined the use of inpatient diagnostic imaging and image-guided procedures to estimate cumulative radiation exposure, radiation exposure based on imaging modality and found that in 200 patients who underwent 2751 imaging studies (79.3\% radiographs, 9.7\% CT, 6.1\% ultrasound, 2.5\% interventional radiology, 2.2\% MRI, $0.4 \%$ nuclear medicine), the mean dose estimate per patient was 14.8 milliSieverts (mSv) with a range of 0 to 130.5 $\mathrm{mSv}$ and the mean cumulative dose estimates were significantly higher for patients whose hospitalizations included time in an ICU $(17.9 \mathrm{mSv}$ versus $11.3 \mathrm{mSv}[p=.01])$ and concluded that majority (82.1\%) of inpatient radiation exposure was attributable to computed tomography.

The technical challenges of IR technique are the efficient use of advanced reconstruction algorithms to enable equivalent diagnostic image quality at a fraction of the dose and overcome the inherent challenges of low-dose scanning ${ }^{[1]}$. These techniques should be designed to seamlessly integrate into routine CT workflow, providing the look and feel of conventional higher dose images ${ }^{[1]}$. These should allow the radiologist/technologist to personalize the image quality based on individual patient need. He should be able to dial in his choice between significantly improving image resolution by using the full radiation dose or scan at a significantly low dose while maintaining the same image quality ${ }^{[1]}$.

The IR techniques use the raw data output from the detector to determine and remove noise and artifacts resulting from low-dose scanning ${ }^{[1]}$. Hence, the initial challenge is to create the necessary speed to make these practical to incorporate into routine clinical world. Typically, most modern scanners are able to generate 20-40 images per second using FBP. The 
scanners using IR techniques could initially generate 1-2 images per second, but with rapid and focused advances, the latest scanners can compete with the FBP scanners and generate approximately 20 images per second.

These challenges have resulted in hardware advances designed specifically to satisfy the performance requirements and processing power needed for low-dose scanning with ease to balance image quality, dose reduction and full dose appearance ${ }^{[1]}$. The core of such iterative work flow is based on automatically generating statistical noise and anatomic structure models to improve operational efficiency. A comparative dual model (noise and anatomic) technique removes noise while preserving morphologic information ${ }^{[1]}$.

In our study, the marked variation of CTDI and DLP values within each anatomical is primarily attributed to the variation in anatomical size, age \& gender of patients ranging from very thin structured patients to markedly obese bariatric patients. The efficiency of dose modulation features as described earlier (ACS, DoseRight angular modulations techniques and Z-modulations) caused to either increase the predefined dose parameters for heavy patients or reduced the dose for patients with lower BMI.

For comparing with typical effective dose values for various anatomical region, as many clinical references described a range of dose per examination, the approach what is taken in this study is to compare with the average value of typical effective dose to the average value of dose analyzed in the patient population analyzed.

Through our dose evaluation and analysis study, we have concluded that the effective radiation dose reduction achieved with use of iterative reconstructions technique is significant. With the radiation dose profile data analyzed for 986 patients, the average dose savings for the thorax examinations were estimated as 33.2\%, for Abdomen and Pelvis scans $-32.455 \%$ and for combined thorax, abdomen \& pelvis (trunk) studies, the dose reduction achieved is $49.706 \%$ in comparison with typical average reported radiation dose values. Average CTDI is found to be lower in trunk examinations of the entire thorax, abdomen and pelvis than the average CTDI value of abdomen \& pelvis studies.

In our study the mean DLP (286.3 mGy.cm) for CT of chest was significantly lower than the IAEA-recommended value of $455 \mathrm{mGy} . \mathrm{cm}^{[7]}$ and the values reported by Papadimitriou et al. - $429 \mathrm{mGy} . \mathrm{cm}$ for centres in Greece and $483 \mathrm{mGy} . \mathrm{cm}$ for centres in Italy ${ }^{[8]}$. The DRL DLP in European guidelines for chest CT is $650 \mathrm{mGy} . \mathrm{cm}$, which is approximately $56 \%$ higher than the corresponding value in our study.

The average CTDI value (7.42 mGy) in our study was lower than that reported by IAEA $16.2 \mathrm{mGy}{ }^{[7]}$ and that by Papadimitriou et al. $(21 \mathrm{mGy}){ }^{[8]}$. Torp et al. ${ }^{[9]}$ reported results from five countries in the Nordic region in which the weighted CTDI values ranged from 10.5 to $11.7 \mathrm{mGy}$. The scanners in that study were helical units developed during the past decade, but no details about the technical factors were available. Hidajat et al. ${ }^{[10]}$ reported results from a study that involved conventional and helical scanners and compared their results with those of other authors ${ }^{[11,12]}$. The range of values presented in that comparison was $13 \mathrm{mGy}-22 \mathrm{mGy}$, with the lower values reported for helical chest CT. Fridberg et al. ${ }^{[13]}$ reported values that were obtained by using both transverse scanners manufactured before 1995 and single-section helical and multi- detector row scanners manufactured after 1995.

The mean weighted CT dose index for abdominal CT was $7.42 \mathrm{mGy}$, which is less than that reported by Hidajat $\mathrm{N}$ et al. $(16.7 \mathrm{mGy})^{[10]}$, Tsapaki V et al. $(10.4 \mathrm{mGy})^{[12]}$ and that by Shrimpton PC et al. $(16 \mathrm{mGy})^{[14]}$. On the other hand, the mean DLP for abdominal CT (495.5 mGy.cm) is similar to the values reported by Papadimitriou et al. (493 mGy.cm - 551 mGy.cm ${ }^{[8]}$. But in this study their values were based on CT scan of upper abdomen only and thus the region scanned was small.

\section{References}

[1] iDose4 iterative reconstruction technique-Philips white paper publication. 
[2] The measurement, reporting, and management of radiation dose in CT: Report of AAPM Task Group 23 of the Diagnostic Imaging Council CT Committee. January 2008.

[3] European guidelines for quality Criteria in Computed Tomography; EUR 16262 EN.

[4] Rehani M, Berry M. Radiation doses in computed tomography [editorial]. BMJ. 2000; 320: 593-594. http://dx.doi.org/10.1136/bmj.320.7235.593

[5] Amy K. Hara, Robert G. Paden, Alvin C. Silva, Jennifer L. Kujak, Holly J. Lawder, William Pavlicek. Iterative Reconstruction Technique for Reducing Body Radiation Dose at CT: Feasibility Study. Am. J. Roentgenol. Sep 2009; 193: 764-771. PMid: 19696291. http://dx.doi.org/10.2214/AJR.09.2397

[6] Lutterman AC, Moreno CC, Mittal PK, Kang J, Applegate KE. Cumulative Radiation Exposure Estimates of Hospitalized Patients from Radiological Imaging.

[7] International Atomic Energy Agency. Optimisation of the radiological protection of patients undergoing radiography, fluoroscopy and computed tomography. Document no. IAEA-TECDOC-1423. Vienna, Austria: International Atomic Energy Agency. 2004.

[8] Papadimitriou D, Perris A, Manetou A, et al. A survey of 14 computed tomography scanners in Greece and 32 scanners in Italy: examination frequencies, dose reference values, effective doses and doses to organs. Radiat Prot Dosimetry. 2003; 104(1): 47-53. PMid: 12862243. http://dx.doi.org/10.1093/oxfordjournals.rpd.a006162

[9] Torp C, Olerud H, Einarson G, Gron P, Leitz W, Servomaa A. Use of the EC quality criteria as a common method of inspecting CT laboratories: a pilot study by the Nordic Radiation Protection Authorities-radiological protection of patients in diagnostic and interventional radiology, nuclear medicine and radiotherapy. Document IAEA-CN-85-175.Vienna, Austria: International Atomic Energy Agency. 2001.

[10] Hidajat N, Wolf M, Nunnemann A, et al. Survey of conventional and spiral CT doses.Radiology. 2001; 218: 395-401. PMid: 11161152. http://dx.doi.org/10.1148/radiology.218.2.r01ja12395

[11] Scheck RJ, Coppenrath EM, Kellner MW, et al. Radiation dose and image quality in spiral computed tomography: multicentre evaluation at six institutions. Br J Radiol. 1998; 71: 734-744. PMid: 9771384.

[12] Tsapaki V, Aldrich JE, Sharma R, Staniszewska MA, Krisanachinda A, Rehani M, et al. Dose reduction in CT while maintaining diagnostic confidence: diagnostic reference levels at routine head, chest and abdominal CT-IAEA-coordinated research project.

[13] Fridberg E, Borretzen I, Olerud M. Dual and multidetector-row CT: what about the doses? Presented at the Radiation Protection Symposium of the North West European Radiation Protection Societies. Utrecht, the Netherlands. June 2-5, 2003.

[14] Shrimpton PC, Hillier MC, Lewes MA, Dunn M. National Radiological Protection Board (NRPB): doses from computed tomography examinations in the UK-2003 review. Document NRPB-W67. Chilton, England: National Radiological Protection Board. 2005. 